\title{
Significant differences in the quality of incident reports - a comparison of four acute hospitals in Finland
}

\author{
Tuula Saarikoski ( $\square$ tuula.saarikoski@ksshp.fi) \\ Central Finland Hospital Nova \\ Kaisa Haatainen \\ Kuopio University Hospital \\ Risto Roine LKT \\ University of Eastern Finland \\ Hannele Turunen \\ University of Eastern Finland
}

\section{Research Article}

Keywords: hospital incident reporting, near miss, incidents, adverse event

Posted Date: December 27th, 2021

DOI: https://doi.org/10.21203/rs.3.rs-1169690/v1

License: (a) (1) This work is licensed under a Creative Commons Attribution 4.0 International License. Read Full License 


\section{Abstract}

\section{Objectives}

The aim of the study was to compare the quality of the content of patient safety incident reports of "near miss" and "adverse event" occurrences, and to examine whether the contributing factors behind the incident were identified.

\section{Methods}

Data were collected from an electronic incident reporting system for a one-year period (2015) at four acute hospitals in Finland. The analysis framework was based on the incident reporting guidelines, and the results were analyzed using statistical methods.

\section{Results}

The most deficiencies were in records of the consequences of the event for the staff and unit (47\%) and the consequences of the event (35\%). The description of the content of "near miss" situations did not differ significantly from "adverse event" situations, but statistically significant differences were found between the hospitals in the quality of the description of the content of incident reports.

\section{Conclusion}

Incident reports did not always identify the processes behind the incident or the factors that contributed to the occurrence of the incident, such as human error. Blaming was still evident in the incident report descriptions.

\section{Introduction}

Patient safety incidents are classified as either a "near miss" or "adverse event" ${ }^{1}$. This study examined the quality of the content of incident reports for these two types of incidents.

In patient care, adverse events are not uncommon, but not all "adverse events" or "near misses" are reported by staff ${ }^{2-4}$. One explanation may be the negative attitude toward reporting and the fear of guilt ${ }^{3,5}$. According to previous studies, the system for reporting is considered to be complex ${ }^{5}$ and difficult to report ${ }^{6}$. The most commonly used system for reporting incidents is electronic patient safety event reporting ${ }^{7}$. Physicians report fewer incidents than nursing staff ${ }^{7}$, which may be due to a misunderstanding that only nurses can make reports ${ }^{8}$ or ignorance concerning who is obliged to report ${ }^{3}$. In addition, physician training may lack guidance on how to make incident reports ${ }^{9}$.

Previous studies have provided information on the reporting of pharmacotherapy-related ${ }^{10,11}$ and fall-related incidents in patients ${ }^{12}$. The role of human error in incidents has been studied previously as part of patient safety culture ${ }^{13}$. Additionally, factors behind human error ${ }^{14}$, e.g., in the implementation of pharmacotherapy ${ }^{15}$ and as a background variables ${ }^{16,17}$ in the case of adverse events, have been investigated.

Comparison of the reporting activity for different types of incidents has found that fewer "near misses" are reported than " adverse events" ${ }^{18}$. A comparative study between different countries also identified differences in how the incident reporting system is used ${ }^{19}$, e.g., whether incidents are reported anonymously, how systematically events are analyzed and how development measures are implemented.

Moreover, patient safety incidents often incur additional $\operatorname{costs}^{20}$. The ultimate purpose of reporting incidents is to prevent them from happening again by learning from them at an individual and system level of the organization. Therefore, from a management and effectiveness perspective, incident reporting should also be combined with cost-effectiveness ${ }^{5}$ and incident learning ${ }^{21,22}$. According to Sengen et al. ${ }^{23}$, a learning organization is based on five different aspects: individual learning, individuals' own operating models, shared vision, team learning, and systems thinking, such as the contributing factors behind incidents.

The learning organization includes knowledge of staff roles and responsibilities ${ }^{24}$. A study has suggested that at the individual and group level, incidents can be learned through colleagues' experiences and by discussion of patient safety with colleagues ${ }^{6}$. According 
to Rashed et al. ${ }^{3}$, it is important to report all types of incidents and reporting systems can be used to identify and learn from errors.

To the best of our knowledge, few previous studies have been conducted on this topic. Thus, this study examined the extent to which incident reports contain the elements described in the instructions for completing the report and analyzed the quality of incident reports. The study supports the objectives of Finland's national patient safety strategy ${ }^{25}$.

The aim of this study was to compare the quality of the content of incident reports for "near miss" reports and "adverse event" occurrences and to determine whether the contributing factors behind the incident were identified. We hypothesized that the nature of the event was not associated with how the occurrence of the incident was described. The hypothesis tested when comparing the data was that there was no difference between hospitals in the description and quality of incident reports.

Four research questions were stated:

1. Is there a difference in the quality of the description of the content of "near miss" and "adverse event" reports?

2. Have the contributing factors related to the incident been described?

3. What percentage of the incident reports led to development actions?

4. Is there a difference in the quality of the description of the content of incidents between the four studied acute care hospitals?

\section{Methods}

The data consisted of incident reports related to patient care made by staff at four acute hospitals in Finland. From the beginning of March 2015, 200 consecutive notifications were included from each hospital (total $N=800$ ). Patient reports and reports on social care, primary health care and hospital schools were excluded from the material. Thus, the final data included 739 notifications.

A framework was used for the analysis based on the instructions of the incident reporting system ${ }^{26}$ supplier on how to complete the notification (20 criteria). The criteria consisted of the nature of the event, the state of handling of the event, the description of the content of the incident, the type of event and the investigator's assessment. The classification was sufficiently, partially or not at all used in the description of the incidents. The analysis framework was peer-reviewed prior to use. In the peer review, 20 cases were analyzed by two patient safety experts according to the analysis cluster, and the consistency of the evaluations made by the evaluators was calculated to be $86 \%$.

In the analysis of the actual study data, the categories "partially" and "not at all" were combined into a single category labelled "no" and the category "sufficient" into the category "yes" because some incident reports contained less than five entries.

The quality of the description of each incident report was analyzed. The adequacy of the content of the incident report was assessed based on how the report had been recorded, what had happened and what the consequences were.

The data were also used to assess the quality of the content of the notification, what could have caused the incident and what were the contributing factors.

The following scale was used to classify the adequacy of the content of incident reports: five or more criteria described in the notification (i.e., $75 \%$ or more described $=$ content described well), three to five criteria described $(50-74 \%=$ content described moderately) and less than three criteria described (<50\% = poor content description) ${ }^{27}$.

IBM SPSS 26 software was used for data analysis using cross-tabulation and Pearson's chi-square test.

The study was approved by the University Ethics Committee, and research permission for each hospital under study was obtained.

\section{Results}

The nature of the event ("near miss" or "adverse event") was used as the background variable. Altogether, $42 \%$ of incident reports were categorized as a "near miss". 
The incident reports were examined to identify whether the notification was made in accordance with the system supplier's instructions on what to record in the notification for three variables (what happened, what was being done, how the incident occurred). The circumstances at the time of the event in the event description was well recorded (over 75\%) in both the "near miss" and "adverse event" reports. There was a good description of what and where the event happened, what was being done and the circumstances at the time of the incident.

For the other four variables (how the situation was handled, what were the consequences for the patient, properly recorded suggestions on how the event could be prevented from recurring, what were the consequences for the staff and unit), entries were rated as moderate (50-74\%). A more detailed analysis of the adequacy of the content relative to the nature of the event was conducted using cross-tabulation and Pearson's chi-square test. The results showed that there was a significant relationship between the nature of the incident ("near miss" or "adverse event") and the incident description "what happened" and "how the incident occurred" ( $\chi 2(2)=3.891 ; p=0.049)$.

A similar significant relationship was observed between the conditions at the time of the event and other factors that, in the opinion of the notifier, contributed to the occurrence of the event $(\chi 2(2)=6.030 ; p=0.014)$. A more detailed description is given in Table 1 .

Table 1

Adequacy of incident content in relation to the nature of the event (occurred to the patient and kept close to the event) expressed as a percentage $(n=739)$

\begin{tabular}{|c|c|c|c|}
\hline Description of the incident & $\begin{array}{l}\text { Adverse } \\
\text { event } \\
n=431\end{array}$ & $\begin{array}{l}\text { Near } \\
\text { miss } \\
n=308\end{array}$ & $\begin{array}{l}\text { Pearson chi- } \\
\text { square value } \\
p\end{array}$ \\
\hline \multirow[t]{2}{*}{ What and how the event happened? } & $95 \%$ & $91 \%$ & 3.891 \\
\hline & & & 0.049 \\
\hline \multirow[t]{2}{*}{ What was being done? } & $86 \%$ & $81 \%$ & 3.150 \\
\hline & & & 0.076 \\
\hline \multirow{2}{*}{$\begin{array}{l}\text { The circumstances at the time of the event and other factors which, in the opinion of the } \\
\text { notifier, contributed to the occurrence of the event. }\end{array}$} & $82 \%$ & $75 \%$ & 6.030 \\
\hline & & & 0.014 \\
\hline \multirow[t]{2}{*}{ How the situation was handled. } & $68 \%$ & $73 \%$ & 1.575 \\
\hline & & & 0.209 \\
\hline \multirow[t]{2}{*}{ What were the consequences for the patient? } & $66 \%$ & $63 \%$ & 0.943 \\
\hline & & & 0.332 \\
\hline \multirow[t]{2}{*}{ Properly recorded suggestions on how the event could be prevented from recurring. } & $59 \%$ & $62 \%$ & 0.609 \\
\hline & & & 0.435 \\
\hline \multirow[t]{2}{*}{ What were the consequences for the staff and unit? } & $51 \%$ & $56 \%$ & 1.758 \\
\hline & & & 0.185 \\
\hline$p<0.05$ & & & \\
\hline
\end{tabular}

Here Table 1

Most incident reports (59\%) included the notifier's suggestions on how to prevent a recurrence. Contributing factors were identified in $79 \%$ of all notifications. As a remedy, care and attention were proposed in $11 \%$ of all notifications. Among the factors that contributed to the incident were inadequate reporting $(10 \%)$, inadequate patient identification $(6 \%)$, insufficient resources $(3 \%)$ and problems with information systems (3\%). A small proportion of incident reports (6\%) blamed someone or some people for the event.

The incident reports also examined whether the incident was recorded as an anomaly related to the operational process or information flow, or was attributed to human error. Based on the results, the recording of human errors was low. The comparison results are shown in Table 2. 
Table 2

Identification of the underlying variables reported by a healthcare professional and evaluated from the researcher's point of view $(n=739)$

\begin{tabular}{|lll|}
\hline & Healthcare professional & Researcher \\
\hline This is an error due to incomplete / incorrect information & $59 \%$ & $61 \%$ \\
\hline This is a process error & $10 \%$ & $36 \%$ \\
\hline Human error in the background & $11 \%$ & $28 \%$ \\
\hline
\end{tabular}

Here Table 2

Less than half $(38 \%)$ of the incident reports led to development measures. The highest number of development measures was observed in Hospital B (54\%) and the lowest in Hospital A (27\%). Among the development measures, changes in operating methods and renewal of guidelines were mentioned.

It was also investigated whether the descriptions of incidents differed between the four hospitals. All the hospitals had a good record (over 75\%) of what was done and what had happened, whereas recording of the treatment of situations and consequences for patients were moderate (50-74\%). In other event categories, the quality of records varied between hospitals. Poor records (less than $50 \%$ ) of how the incident had affected the staff were found in Hospitals B and D

Hospital A had the fewest records of what was being done, and in this respect the hospitals also differed statistically significantly ( $p=$ 0.014). Descriptions of what had happened in the emergency were best recorded in Hospital $B$, and in this too, the hospitals differed statistically significantly $(p=0.001)$. There were also significant differences in the recording of the incident treatment between different hospitals, with the highest number of entries in Hospital $\mathrm{C}(p=0.016)$. There was no statistically significant difference between hospitals in the description of the consequences of the incident, but description of the consequences for the staff and unit in the records differed significantly between the different hospitals. The lowest number of registrations was in Hospital D $(p<0.01)$. The conditions at the time of the event and other factors that contributed to the occurrence of the event were best described in Hospital B and worst in Hospital A. Again, the hospitals differed statistically significantly $(p<0.01)$. Records to prevent recurrence of the incident were best in Hospital B and there was a statistically significant difference between hospitals $(p=0.05)$. A detailed description is given in Figure 1. Within a single hospital, there was variation between different variables.

Here Figure 1

\section{Discussion}

The nature of an event, i.e., whether it was a near miss event or adverse event, should not affect the reporting activity or event description ${ }^{28}$. Based on this research, it seems that near miss notifications were generally well reported, i.e., they are informative, which is important from the perspective of anticipation and learning.

The factors and circumstances that contribute to each incident should be identified. If near miss notices are carefully reviewed with staff, necessary protection mechanisms can be identified and devised together.

In a learning organization, patient safety and incidents should be reviewed on a daily basis and addressed systematically as part of the development of the patient safety process with staff, organizational management, trust staff and patients ${ }^{6}$. The quality of incident reporting can also be viewed from the perspective of the development of the learning organization ${ }^{29}$. Although the activity of notifications is constantly increasing, the number of development measures taken on the basis of notifications is small ${ }^{22,30,31}$. It is the responsibility of staff to report any "near miss" events related to a patient's care and any "adverse event" that has occurred to the patient. Supervisors should process the notifications and plan development measures together with the staff. Development measures proposed by staff, such as increased accuracy or diligence, are difficult to implement without addressing why accuracy was inadequate during the event and what factors contributed to it. It is important that incident reports are processed and learned from together ${ }^{28}$. 
Incident reports do not necessarily identify the practices and related processes that contribute to the occurrence of an event, such as patient identification or the different stages of medication implementation ${ }^{32}$. However, identifying them could help to improve development measures and assist the supervisor in analyzing the reports The processes behind an incident usually involve different professional groups and may be related to, e.g., the flow of information and its shortcomings ${ }^{32}$. When examining the quality of a notification, the root causes behind the occurrence of an incident are often left unidentified, even though they contribute to a deeper analysis of the occurrence of the incident and to consideration of how incidents could be prevented, e.g., through checklists or training 4 .

The incident reporting system needs to output information to support management quickly in a user-friendly manner ${ }^{5,33}$. In addition, it should include a variety of analytical tools to study learning processes ${ }^{28}$. Analysis tools are needed when dealing with different stages of processes and the course of an event. The system should also notify if the descriptions of the event are insufficient ${ }^{7}$. If the system were connected to the patient information system, an incident notification ${ }^{34}$ could be made automatically in the event of an incident, but this could be challenging in terms of data protection, security and anonymity. According to the results, guilt and blaming is still reflected in incident reports. However, the aim is that staff should also be able to report incidents without fear of being blamed $3,5,35$

The four studied hospital had the same incident reporting system in place. Thus, the underlying instructions for completing the report were the same. There were qualitative differences in the incident reporting between hospitals but also within them. There were shortcomings in identifying human error. If underlying human factors can be identified, they can be addressed, e.g., by looking at job descriptions or the content of shifts, as well as the adequacy of human resources. Patient safety culture has an impact on how human error is reported ${ }^{13}$, as reporting and filling in reports may be influenced by the different organizational culture of hospitals ${ }^{36}$.

\section{Conclusion}

The quality of the descriptions in incident reports differed between hospitals. Notifications did not always identify the factors or the underlying processes that contributed to the occurrence of the event. Deficiencies in identifying the contributing factors suggest a need for additional training and guidance in utilizing the patient safety incident system. This could improve reporting activity and eliminate under-reporting problems. The incident reporting system should be easy to use and provide analytical information to support management.

Currently, head nurses, head physicians and experts in various fields have been named as handlers of patient safety incident reports in hospitals. As a result, notification processing is often decentralized, which poses challenges for consistent processing. One solution in hospitals could be the centralized processing of reports, which could focus on the quality and consistency of processing to help identify risks associated with patient care and necessary development measures.

\section{References}

1. WHO. Patient safety incident reporting and learning systems: Technical report and guidance. 2020. https://www.who.int/publications/i/item/9789240010338.

2. Härkänen M, Paananen J, Murrells T, Rafferty AM, Franklin BD. Identifying risks areas related to medication administrations - text mining analysis using free-text descriptions of incident reports. BMC Health Serv Res. 2019;19(1):791. doi:10.1186/s12913-0194597-9

3. Rashed A, Hamdan M. Physicians' and nurses' perceptions of and attitudes toward incident reporting in Palestinian hospitals. $J$ Patient Saf. 2019;15(3). doi:10.1097/PTS.0000000000000218

4. Eiding $\mathrm{H}$, Røise $\mathrm{O}$, Kongsgaard UE. Potentially severe incidents during interhospital transport of critically ill patients, frequently occurring but rarely reported: A prospective study. J Patient Saf. 9000;Publish Ah. https://journals.Iww.com/journalpatientsafety/Fulltext/9000/Potentially_Severe_Incidents_During_Interhospital.99094.aspx.

5. Carlfjord S, Öhrn A, Gunnarsson A. Experiences from ten years of incident reporting in health care: A qualitative study among department managers and coordinators. BMC Health Serv Res. 2018;18(1):113. doi:10.1186/s12913-018-2876-5

6. Sujan M. An organisation without a memory: A qualitative study of hospital staff perceptions on reporting and organisational learning for patient safety. Reliab Eng Syst Saf. 2015;144:45-52. doi:https://doi.org/10.1016/j.ress.2015.07.011

Page 6/9 
7. Gong Y, Kang H, Wu X, Hua L. Enhancing patient safety event reporting: A systematic review of system design features. App/ Clin Inf. 2017;8:893-909. doi:10.4338/ACl-2016-02-R-0023

8. Varallo FR, Passos AC, Nadai TR de, et al. Incidents reporting: Barriers and strategies to promote safety culture. Rev da EsC Enferm da USP. 2018;52(0). doi:10.1590/s1980-220x2017026403346

9. Louis MY, Hussain LR, Dhanraj DN, et al. Improving patient safety event reporting among residents and teaching faculty. Ochsner J. 2016;16(1):73-80. https://pubmed.ncbi.nlm.nih.gov/27046410.

10. Westbrook JI, Li L, Lehnbom EC, et al. What are incident reports telling us? A comparative study at two Australian hospitals of medication errors identified at audit, detected by staff and reported to an incident system. Int J Qual Heal Care. 2015;27(1). doi:10.1093/intqhc/mzu098

11. Härkänen M, Turunen H, Vehviläinen-Julkunen K. Differences between methods of detecting medication errors: A secondary analysis of medication administration errors using incident reports, the Global Trigger Tool method, and observations. $J$ Patient Saf. 9000;Publish Ah.

https://journals.Iww.com/journalpatientsafety/Fulltext/publishahead/Differences_Between_Methods_of_Detecting.99602.aspx.

12. Richardson A, Carter R. Falls in critical care: A local review to identify incidence and risk. Nurs Crit Care. 2017;22(5):270-275. doi: $10.1111 /$ nicc. 12151

13. Duarte S da CM, Stipp MAC, Cardoso MMVN, Büscher A. Patient safety: Understanding human error in intensive nursing care. Rev Esc Enferm USP. 2018;52:e03406. doi:10.1590/S1980-220X2017042203406

14. Chatziioannidis I, Mitsiakos G, Vouzas F. Focusing on patient safety in the neonatal intensive care unit environment. $J$ Pediatr Neonatal Individ Med. 2017;6(1):e060132. doi:10.7363/060132

15. Hsieh M-C, Chiang P-Y, Wang EM-Y, et al. The investigation of human error analysis in adverse drug events in Taiwan - From the perspective of causality assessment. Hum Factors Ergon Manuf Serv Ind. 2019;29(4):340-349. doi:10.1002/hfm.20791

16. Sendlhofer G, Schweppe P, Sprincnik U, et al. Deployment of critical incident reporting system (CIRS) in public Styrian hospitals: A five year perspective. BMC Health Serv Res. 2019;19(1):412. doi:10.1186/s12913-019-4265-0

17. Hu W, Carver J, Anu V, Walia G, Bradshaw G. Using human error information for error prevention. Empir Softw Eng. toukokuuta 2018. doi:10.1007/s10664-018-9623-8

18. Espin S, Carter C, Janes N, McAllister M. Exploring health care professionals' perceptions of incidents and incident reporting in rehabilitation settings. J Patient Saf. 2019;15(2).

https://journals.Iww.com/journalpatientsafety/Fulltext/2019/06000/Exploring_Health_Care_Professionals_Perceptions.12.aspx.

19. Manser T, Imhof M, Lessing C, Briner M. A cross-national comparison of incident reporting systems implemented in German and Swiss hospitals. Int J Qual Heal Care. 2017;29(3):349-359. doi:10.1093/intqhc/mzx030

20. Wulff Risør B, Lisby M, Sørensen J. Cost-effectiveness analysis of an automated medication system implemented in a Danish hospital setting. Value Heal. 2017. doi:10.1016/j.jval.2017.03.001

21. Stavropoulou C, Doherty C, Tosey P. How effective are incident-reporting systems for improving patient safety? A systematic literature review. Milbank Q. 2015;93(4). doi:10.1111/1468-0009.12166

22. Macrae C. The problem with incident reporting. BMJ Qual Saf. 2016;25(2). doi:10.1136/bmjqs-2015-004732

23. Peter M. Senge, Art Kleiner, Charlotte Roberts, Richard B. Ross BJS. The fifth discipline fieldbook Strategies and tool for building a learning organization. 1994.

24. Lalani M, Bussu S, Marshall M. Understanding integrated care at the frontline using organisational learning theory: A participatory evaluation of multi-professional teams in East London. Soc Sci Med. 2020;262:113254. doi:https://doi.org/10.1016/j.socscimed.2020.113254

25. Sosiaali- ja terveysministeriö. Valtioneuvoston periaatepäätös. Potilas-ja asiakasturvallisuusstrategia 2017-2021. Vsk 2017:9. Helsinki: Sosiaali- ja terveysministeriö; 2017.

26. Awanic Oy. HaiPro. http://awanic.com/tuotteet-ja-palvelut/. Published 2016.

27. Kuosmanen A, Tiihonen J, Repo-Tiihonen E, Eronen M, Turunen H. Patient safety culture in two Finnish state-run forensic psychiatric hospitals. J Forensic Nurs. 2013;9(4).

https://journals.Iww.com/forensicnursing/Fulltext/2013/10000/Patient_Safety_Culture_in_Two_Finnish_State_Run.6.aspx. 
28. Hewitt T, Chreim S, Forster A. Incident reporting systems: A comparative study of two hospital divisions. Arch Public Health. 2016;74:34. doi:10.1186/s13690-016-0146-8

29. Leistikow I, Mulder S, Vesseur J, Robben P. Learning from incidents in healthcare: The journey, not the arrival, matters. BMJ Qual Saf. 2017;26(3):252 LP - 256. doi:10.1136/bmjqs-2015-004853

30. Liukka M, Hupli M, Turunen H. Problems with incident reporting: Reports lead rarely to recommendations. J Clin Nurs. 2019;28(910):1607-1613. doi:10.1111/jocn.14765

31. Ahlberg EL, Elfstrom J, Borgstedt MR, et al. Learning from incident reporting? Analysis of incidents resulting in patient injuries in a web-based system in Swedish health care. J Patient Saf. 2017. doi:10.1097/PTS.0000000000000343 [doi]

32. Aaronson EL, Brown D, Benzer T, Natsui S, Mort E. Incident reporting in emergency medicine: A thematic analysis of events. $J$ Patient Saf. 2019;15(4).

https://journals.Iww.com/journalpatientsafety/Fulltext/2019/12000/Incident_Reporting_in_Emergency_Medicine_A.34.aspx.

33. Denecke K. Automatic Analysis of critical incident reports: Requirements and use cases. Stud Health Technol Inform. 2016;223:85-92.

34. Mitchell I, Schuster A, Smith K, Pronovost P, Wu A. Patient safety incident reporting: A qualitative study of thoughts and perceptions of experts 15 years after \{\textquoteleft\}To Err is Human\{\textquoteright\}. BMJ Qual Saf. 2016;25(2):92-99. doi:10.1136/bmjqs-2015-004405

35. Mjadu TM, Jarvis MA. Patients' safety in adult ICUs: Registered nurses' attitudes to critical incident reporting. Int J Africa Nurs Sci. 2018;9:81-86. doi:https://doi.org/10.1016/j.ijans.2018.09.001

36. Burlison JD, Quillivan RR, Kath LM, et al. A multilevel analysis of U.S. hospital patient safety culture relationships with perceptions of voluntary event reporting. J Patient Saf. 2020;16(3).

https://journals.Iww.com/journalpatientsafety/Fulltext/2020/09000/A_Multilevel_Analysis_of_U_S_Hospital_Patient.2.aspx.

\section{Figures}




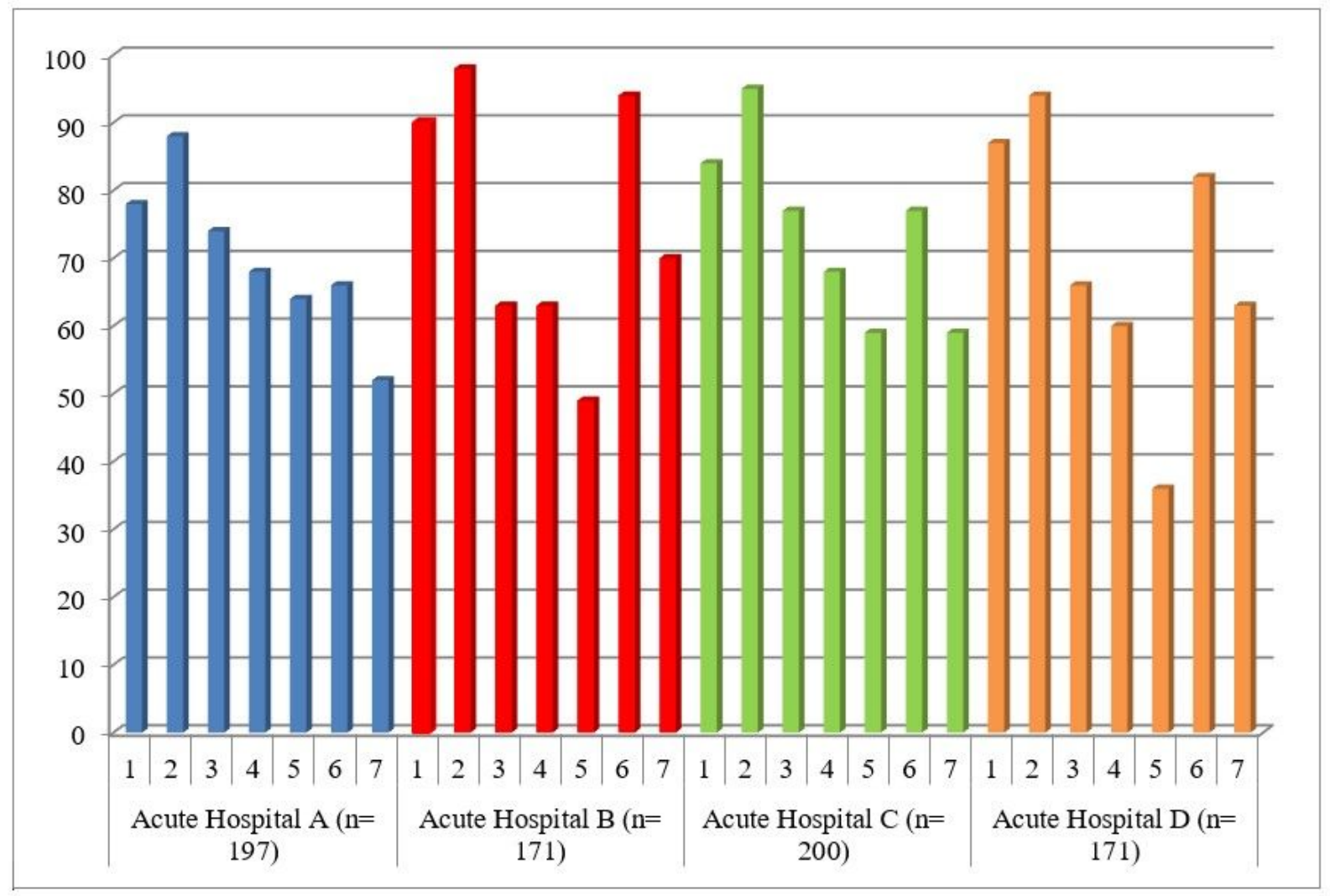

$1=$ What was being done $(\mathrm{x} 2=10.625, \mathrm{df}=3, p=0.014)$

$2=$ What happened and how $(\mathrm{x} 2=15.478, \mathrm{df}=3, p=0.001)$

$3=$ How was the situation handled $(\mathrm{x} 2=10.331, \mathrm{df}=3, p=0.016)$

$4=$ What were the consequences for the patient $(\mathrm{x} 2=3.885, \mathrm{df}=3, \boldsymbol{p}=0.274)$

$5=$ What were the consequences for the staff and unit $(\mathrm{x} 2=33,965, \mathrm{df}=3, p<0.01)$

$6=$ Circumstances at the time of the event and other factors which, in the opinion of the notifier, influenced the occurrence of the event $(\mathrm{x} 2=45.408, \mathrm{df}=3, p<0.01)$

$7=$ Properly recorded suggestions on how to prevent recurrence of the event $(\mathrm{x} 2=12.659, \mathrm{df}=3, \boldsymbol{p}=0.05)$

\section{Figure 1}

Comparison between investigated hospitals of classified incident descriptions (\%). 